\title{
A man with drug-induced psychosis attempts to swallow his cellular phone
}

\author{
Zachary Levy $\cdot$ John Jesus $\cdot$ Arayel Osborne • \\ Patrick Matthews
}

Received: 30 January 2013/Accepted: 20 April 2013/Published online: 5 May 2013

(C) SIMI 2013

A 35 year-old man with no significant past medical history presented to the emergency department (ED) after abusing phencyclidine (PCP). Responding to command auditory hallucinations, he attempted to swallow his $4 \mathrm{~cm} \times 8 \mathrm{~cm}$ smartphone. On arrival, he was agitated but alert, handling his secretions poorly and in moderate respiratory distress. An electronic device was clearly protruding from his oropharynx. He was tachypneic (24 respirations/min) with an oxygen saturation of $92 \%$ on room air, a heart rate of 131 beats per minute and a blood pressure of 137/64 mmHg.

Emergency physicians immediately attempted to remove the device with Magill forceps, but were unsuccessful. A "trauma code" was announced bringing a surgical intensivist, an anesthesiologist, and appropriate nursing staff to the bedside, while simultaneously indicating that an operating room (OR) should be prepared. The patient was emergently transported to the OR and "double prepped" for both emergent cricothyrotomy and oropharyngeal intubation. The device was successfully removed under procedural sedation without the need for surgical intervention. A mucosal tear was noted in the posterior pharynx. The patient became markedly agitated post-procedure, and, given the recent trauma, was intubated for airway protection. He subsequently developed bilateral pneumothoraces and significant subcutaneous emphysema throughout his neck and upper torso.

Diagnosis: Posterior pharyngeal rupture and bilateral pneumothoraces following attempted foreign body ingestion (Fig. 1). Computerized tomography of the neck and chest revealed a defect in the posterior wall of the oropharynx at the C2 level, along with significant mediastinal

Z. Levy $(\bowtie) \cdot$ J. Jesus $\cdot$ A. Osborne $\cdot$ P. Matthews Christiana Care Health System, Newark, DE, USA e-mail: levy.zachary@gmail.com air and right-greater-than-left pneumothoraces. The patient was treated with a right-sided chest tube and was observed in the intensive care unit. He was treated for 7 days with broad-spectrum antibiotics, specifically, piperacillin and tazobactam. He did not require surgical repair of the pharyngeal defect and was discharged from the hospital 2 weeks later.

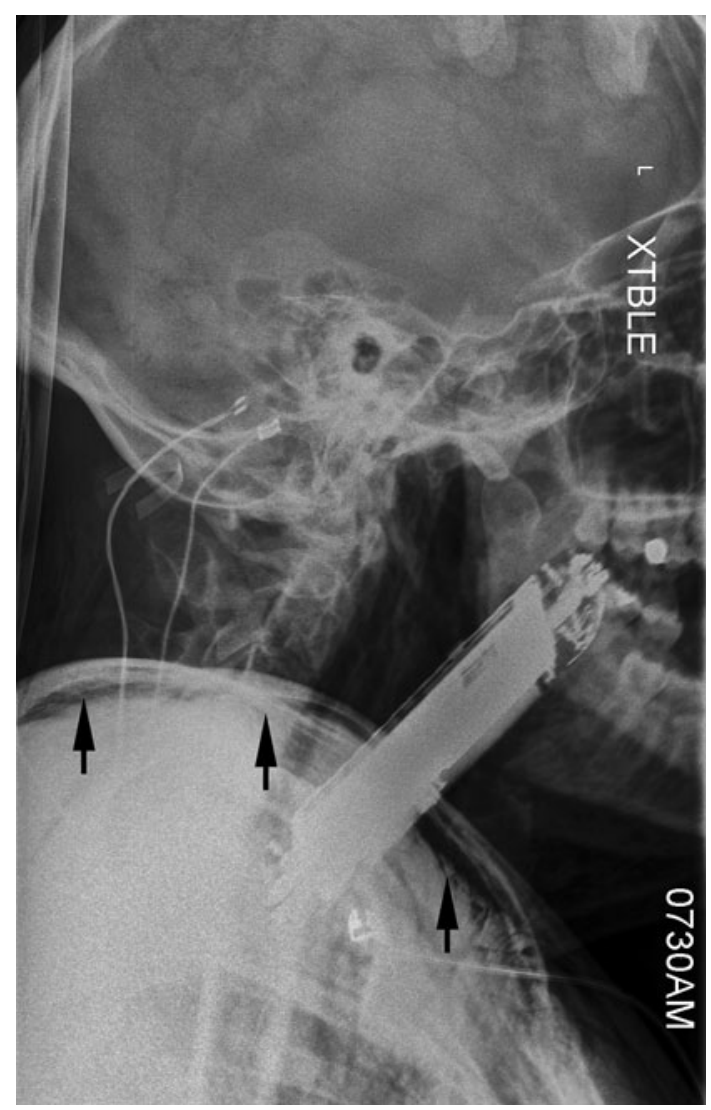

Fig. 1 Lateral neck film 
Pharyngeal perforation usually results not only from iatrogenic injury, but may also be due to foreign body ingestion or barotrauma [1]. Subcutaneous emphysema may be subtle and a delay in diagnosis is possible, carrying a slightly higher mortality compared to cases that are diagnosed early [2]. Treatment varies and may range from immediate surgical repair to conservative management and observation [3]. In addition to establishing an airway, antibiotics are often administered in non-operative cases to prevent abscess formation and mediastinitis; although controversy exists in this use of prophylactic antibiotics owing to how infrequently pharyngeal perforation is encountered [4].

\section{References}

1. Gupta NM, Kaman L (2004) Personal management of 57 consecutive patients with esophageal perforation. Am J Surg 187(1):58-63

2. Reeder LB, DeFilippi VJ, Ferguson MK (1995) Current results of therapy for esophageal perforation. Am J Surg 169(6):615-617

3. Vallbohmer D, Holshcer AH, Holscher M, Bludau M, Glutschow C, Stippel D, Bollschweiler E, Schroder W (2010) Options in the management of esophageal perforation: analysis over a 12-year period. Dis Esophagus 23(3):185-190

4. Smith D, Woolley S (2006) Hypopharyngeal perforation following minor trauma: a case report and literature review. Emerg Med J 23(1):e7

Conflict of interest None. 